\title{
Unilateral nevoid telangiectasia, diagnostic and therapeutic challenge
}

\begin{abstract}
The Unilateral Nevoid Telangiectasia consists of a rare vascular dermatitis whose etiology remains undefined, making it difficult to treat. Due to the scarcity of data in the literature its diagnosis and its treatment represent a challenge. There is a predominance of this pathology in females in the proportion of $2: 1$. The main hypothesis for its etiopathogeny is hyperestrogenism. This justifies the performance of pregnancy tests, dosage of liver enzymes or serologies for hepatotrophic viruses to investigate situations that raise estrogen. The diagnosis is exclusively clinical. The therapeutic modality instituted is laser therapy. Both the Nd: YAG 1064nm long pulse laser and pulsed dye laser are effective for the treatment of vascular lesions.
\end{abstract}

Volume 2 Issue 5 - 2018

\author{
Denise Camilios Cossiolo, Ana Cecília \\ Siqueira Camargo, Rodrigo Eik Sahyun, Maria \\ Fernanda Camargo Boin \\ Catholic University of Paraná, Brazil
}

\author{
Correspondence: Denise Camilios Cossiolo, Medicine \\ Student, Catholic University of Paraná, Av. Jockey Club, 485 \\ Hipica, Londrina - PR, Tel (43) 999462733 \\ Email deniseceossiolo@gmail.com
}

Received: August 20,2018 | Published: October 19, 2018

\section{Introduction}

The Unilateral Nevoid Telangiectasia (UNT) was described by Zeisler and Blaschko in 1899, but its current denomination was defined by Selmanowitz in $1970 .^{1-3}$ The term nevóide refers to the similarity with other congenital cutaneous lesions blaschkoide and does not indicate a specific morphology of telangiectasia. ${ }^{4}$ It is a rare vascular dermatitis defined by areas of superficial telangiectasia with unilateral distribution, arborescent, non-confluent and without central vessel..$^{1,2,5}$ The most accepted etiopathogenesis is associated with high levels of estrogen. ${ }^{1}$ This pathology is classified as congenital and acquired, being the first one more prevalent in men, by autosomal dominant inheritance occurring during or after the neonatal period. The second one has a greater predilection for women during their fertile age and is linked to hyperestrogenism or elevation of vasoactive substances. ${ }^{1,2,6}$

\section{Physiopathology}

The exact etiopathogenesis of Unilateral Nevoid Telangiectasia remains undefined. ${ }^{7,8}$ The main hypothesis is related to high levels of estrogen. ${ }^{1}$ This is explained by the prevalence of the disease in situations of physiological hyperestrogenism (gestation, puberty, contraceptive use, hormonal therapy) and pathological (liver disease, chronic alcoholism with or without cirrhosis). ${ }^{1,29}$ Although there are cases of UNT without significant elevation of estrogen levels and with normal liver function tests. ${ }^{10}$ Endothelial cells have estrogen receptors and this hormone influences angiogenesis. ${ }^{9}$ Vascular endothelial growth factor (VEGF) is linked to the pathogenesis of UNT in patients with underlying liver disease. ${ }^{5}$

In only $20 \%$ is detected a systemic disease that is responsible for the increase of estrogen. In the literature, there is a report that relates UNT to hyperthyroidism, portal hypertension and carcinoid syndrome. ${ }^{7}$ The unilateral lesion is associated with the presence of cells with abnormal estrogen sensitivity, arranged congenitally in the path of the dermatome. There are authors who link the segmental nature of this pathology to mosaicism, originating from somatic mutations of cell populations during embryonic development, which becomes evident in situations of hyperestrogenism. ${ }^{1,2}$ This theory is reinforced when there are other lesions from mosaicism, such as linear inflammatory verrucous epidermal nevus (NEVIL) associated with the condition. Other mechanisms are also studied, such as: hemodynamic disorders, neurological alterations, influence of angiogenic factors and connective tissue disorders. ${ }^{1}$

The genetic mechanism of Twin spotting (or didymosis), specific type of somatic gene recombination with loss of heterozygosity, may explain the coexistence of UNT and Bier stains and Meloratosis. ${ }^{8,11,12}$ This mechanism considers that an embryo initially carries two recessive alleles in the same locus. If Crossing-over occurs on these chromosomes during mitosis, two distinct clones of homozygous daughter cells can be generated. ${ }^{11}$

\section{Epidemiology}

The incidence of UNT is not known to be probably underreported. ${ }^{6}$ Although it is a rare syndrome, the increase in reports in recent years suggests that UNT may be more common than previously. There is a predominance of this pathology in females in the proportion of 2:1. The predominance is verified in many patients who are in the beginning of puberty and during the gestation, by the state of hiperestrogenismo. ${ }^{4}$

\section{Clinical manifestations}

The clinical presentation is a linear arrangement of dilated vessels of small caliber, unilaterally distributed, arborescent, without the presence of a central vessel..$^{2,8,11}$ A pale halo can be observed around telangiectasia. ${ }^{9}$ Its disposition can be linear, segmental or metameric. ${ }^{2}$ From the third to fourth cervical dermatomes and the trigeminal nerve, face, shoulder, arm and upper trunk are the most involved sites, but the lower extremities (lumbar and sacral) may also be affected. ${ }^{6,10}$ Some authors have observed that the metameric distribution usually follows the lines of Blaschko, this could be the result of the migration of cells during embryonic development. ${ }^{2}$

Mazereeuw-Hautier et al. reported an isolated case of pyogenic granuloma that developed on UNT affected skin. This fact is probably a coincidence, however, there is a possibility that the granuloma was manifested due to the vascular physiological disturbance generated by UNT. ${ }^{4}$ The lesions are asymptomatic and there is no systemic or mucosal involvement. Its course is benign and, in most cases, the 
course is chronic and persistent. ${ }^{2}$ There are reports of postpartum spontaneous regression, which suggests a direct relationship between hormonal elevation during pregnancy. ${ }^{2,4}$ Cunliffe et al. selected women who developed UNT during pregnancy but that in the postpartum had full resolution of the condition, in order to confirm the influence of estrogen in the pathogenesis of this disease. Estrogen cream was applied to these women in the areas previously affected by telangiectasias in order to induce a recurrence. However, they did not succeed, suggesting that estrogen is not the only factor responsible for pathology. ${ }^{4}$

\section{Diagnosis}

The diagnosis is exclusively clinical. ${ }^{2}$ In order to define the possible cause of the condition, can carry out pregnancy tests, dosage of liver enzymes or serologies for hepatotrophic viruses. ${ }^{6}$ The diagnosis of telangiectasia can be confirmed by histopathological examination, in which the presence of dilated and thin-walled capillaries in the papillary dermis, without endothelial proliferation or neoangiogenesis, mild perianexial and perivascular lymphocytic infiltrate. ${ }^{2,6,9,13}$ However, it may require a perilesional skin biopsy for comparison. ${ }^{6}$

Its main differential diagnosis is Serpiginous Angioma and in this there is the proliferation of dilated capillaries, with thickened walls in the papillary dermis. ${ }^{1}$ Other possible differential diagnoses are: primary telangiectasia, mainly the linear Atrofodermia of Moulin and secondary telangiectasias, such as Erythema ab igne or use of topical corticosteroids4. In addition, UNT needs to be clinically differentiated from a typical hemangioma. The Nevo Flammeus, on the other hand, presents a more macular appearance. It is present from birth and should be considered when there is suspicion of UNT with congenital etiology. ${ }^{14}$

The Immunohistochemistry may also help differentiate UNT from other dermatological lymphovascular conditions. For example, the D2-40 marker is used to differentiate lymphatic lesions from vascular. In contrast, the GLUT-1, a human erythrocyte glucose transporter, is associated with cutaneous hemangiomas and other vascular malformations. ${ }^{10}$ The Doppler flowmeter laser, which analyzes the microcirculation, is capable of detecting Unilateral Nevoid Telangiectasia in its subclinical phase. It can find a hyperperfusion of the affected regions, evidenced by erythema, an increase in the caliber of the capillaries, a characteristic also present in scleroderma. In the nail capillaroscopy can observe the presence of megacapilares, as well as in some collagenoses.

\section{Treatment}

Both the Nd: YAG $1064 \mathrm{~nm}$ long pulse laser and the pulsed dye laser are effective for the treatment of vascular lesions. ${ }^{7}$ The Nd: YAG $1064 \mathrm{~nm}$ long pulse laser, in turn, is more commonly used for larger vessels. ${ }^{2}$ This type of laser has more effective absorption by the deoxyhemoglobin which is found in greater quantity in the more calibrous veins, instead of the small dilated vessels observed in the UNT. In addition, the Nd: YAG tends to penetrate the deeper dermis, due to the longer wavelength and the lower absorption by surface chromophores. However, in the pathology in question telangiectasias are located in the superficial dermis. ${ }^{7}$ Thus, pulsed dye laser is the most appropriate method for the treatment of nevoid telangiectasia. ${ }^{1,2}$ There is evidence of significant hyperpigmentation and hypopigmentation at the end of treatment. ${ }^{1}$ Other therapeutic modalities described are: electrocoagulation, radiofrequency, cryotherapy, $\mathrm{CO}_{2}$ laser and argon. ${ }^{2}$ In cases of hyperestrogenism linked to systemic diseases, resolution of the pathology may improve the UNT profile. ${ }^{6}$

\section{Conclusion}

Because Unilateral Nevoid Telangiectasia is a rare disease whose etiopathogenesis is not fully elucidated, it needs new research that contributes to its full knowledge. So that, finally, there is an expansion in the diagnostic and therapeutic possibilities.

\section{Acknowledgements}

None.

\section{Conflict of interest}

Author declares that there is no conflict of interest.

\section{References}

1. Jordão JM, Haendchen LC, Berestinas TC, et al. Telangiectasia nevoide unilateral adquirida em homem hígido. An Bras Dermatol. 2010;85(6):912-914

2. Serra M, Reyes V, Hernández M, et al. Telangiectasia nevoide unilateral: reporte de dos casos en mujeres embarazadas. Arch Argent Dermatol. 2012;62:144-147.

3. Shaver CM, Cusack CA. A rare case of congenital unilateral nevoid telangiectasia. J Am Acad Dermatol. 2016;74(5 Supl 1):AB208.

4. Wenson SF, Jan F, Sepehr A. Unilateral nevoid telangiectasia syndrome: a case report and review of the literature. Dermatol Online J. 2011;15;17(5):2

5. Kim HJ, Kim KO, Kim YS, et al. Acquired Unilateral Nevoid Telangiectasia Accompanied by Liver Metastasis of Colon Cancer. Ann Dermatol. 2016;28(3):404-405.

6. Lencastre A, Lopes MJP. Case for diagnosis. An Bras Dermatol. 2013;88(2):299-301.

7. Kim J, Cho SB, Cho S, et al. Unilateral nevoid telangiectasia associated with ipsilateral melorheostosis. Ann Dermatol. 2012;24(2):206-208.

8. Chen LA, Tsai TH, Chang YJ, et al. Unilateral nevoid telangiectasiaresponse to long-pulsed Nd:YAG laser and pulsed dye laser: a case report. Dermatologica Sinica. 2010;28(1):44-45.

9. Kim J, Cho SB, Cho S, et al. Unilateral nevoid telangiectasia associated with ipsilateral melorheostosis. Ann Dermatol. 2012;24(2):206-208.

10. Guedes R, Leite L. Unilateral nevoid telangiectasia: a rare disease? Indian J Dermatol. 2012;57(2):138-140.

11. Smith JÁ, Kamangar F, Prakash N, et al. Unilateral nevoid telangiectasia syndrome (UNTS) associated with chronic hepatitis $\mathrm{C}$ virus and positive immunoreactivity for VEGF. Dermatol Online J. 2014;15;20(6).

12. Pinho A, Gouveia M, Oliveira $\mathrm{H}$, et al. Bier spots and unilateral nevoid telangiectasia: more than just a coincidence. An Bras Dermatol. 2015;90(5):769-770

13. Froes GC, Bittencourt FV, Resende ACB, et al. Caso para diagnostic. An Bras Dermatol. 2008;83(1):90-92.

14. Kawakami T, Kimura S, Soma Y. Unilateral nevoid telangiectasia on the lower extremity of a pediatric patient. J Am Acad Dermatol. 2010;62(3):528-530. 